\title{
Pressure-induced wall thickness variations in multi-layered wall of a pollen tube and Fourier decomposition of growth oscillations
}

\author{
Mariusz Pietruszka and Aleksandra Haduch-Sendecka \\ University of Silesia, Faculty of Biology and Environment Protection, Laboratory of Plant Physiology, Katowice, Poland
}

\begin{abstract}
The augmented growth equation introduced by Ortega is solved for the apical portion of the pollen tube as an oscillating volume, which we approach in the framework of a two-fluid model in which the two fluids represent the constant pressure and the fluctuating features of the system. Based on routine Fourier analysis, we calculate the energy spectrum of the oscillating pollen tube, and discuss the resonant frequency problem of growth rate oscillations. We also outline a descriptive model for cell wall thickness fluctuations associated with small, yet regular variations $(\sim 0.01 \mathrm{MPa})$ observed in turgor pressure. We propose that pressure changes must lead to the sliding of wall layers, indirectly resulting in a wave of polarization of interlayer bonds. We conclude that pollen tube wall thickness may oscillate due to local variations in cell wall properties and relaxation processes. These oscillations become evident because of low amplitude/high frequency pressure fluctuations $\delta P$ being superimposed on turgor pressure $P$. We also show that experimentally determined turgor pressure oscillates in a strict periodical manner. A solitary frequency $f_{0} \approx 0.066 \mathrm{~Hz}$ of these $(\sim 0.01$ MPa in magnitude) oscillations for lily pollen tubes was established by the discrete Fourier transform and Lorentz fit.
\end{abstract}

Key words: Cell wall - Lily - Pressure fluctuations - Osmotic pressure - Resonance frequency - Tobacco - Turgor pressure

\section{Introduction}

All rapid axis extension in plants is based on volumetric growth of individual plant cells, which in turn depends on the interaction between the hydrostatic pressure generated within the cell (turgor pressure) and the extensibility of the wall material itself. This characteristically biophysical mode of growth, which is one of the features that distinguishes the evolution of plants, affords an unique opportunity to examine the control mechanisms underlying morphogenetic ontogeny from a purely mathematical point of view.

The plant cell wall is a complex system of composite materials about $0.1-1 \mu \mathrm{m}$ thick, consisting of cellulose microfibrils embedded in a matrix of hemicelluloses and pectins (Cosgrove 1997). Hemicelluloses serve to anchor the microfibrils in the surrounding matrix by bonding

Correspondence to: Mariusz Pietruszka, University of Silesia, Faculty of Biology and Environment Protection, Laboratory of Plant Physiology, Jagiellońska 28, PL-40032, Katowice, Poland E-mail: mariusz.pietruszka@us.edu.pl non-covalently to their surface (see Fig. 3 in Veytsman and Cosgrove 1998). This hierarchically built structure consists of multiple layers, implying that mechanical deformation of the wall will likely result in the slippage of polymers and the sliding of layers (Geitmann 2010). Understanding the rheological behaviour of the cell wall is therefore crucial for understanding observed changes in cell wall thickness (McKenna et al. 2009). The growth of walled cells is governed largely by the changing mechanical (viscoelastic) properties of the wall as it resists vacuolar turgor pressure to produce a quasi-stable equilibrium. A special example of a growing plant cell is the growth of pollen tubes (Plyushch et al. 1995; Derksen et al. 2011). We note that changes in wall thickness have been observed during pulsatile growth of Gasteria pollen tubes (Plyushch et al. 1995). An important and frequently observed feature of pollen tube elongation is that both the growth in length and its derivative, the growth rate, oscillate. Many of the underlying processes also oscillate with the same period, but usually out of phase (see Fig. 3 in Holdaway-Clarke and Hepler 2003; Fig. 3 in Cardenas et al. 2006; Fig. 4A in Cardenas et al. 2008; Fig. 1C in Zonia and 
Munnik 2011). Time-lapse images of lily pollen tubes reveal that during oscillatory growth the wall thickness at the apex of the cell changes. McKenna et al. (2009) showed that the cell wall thickness oscillates with the same period but out of phase with the oscillations in growth rate.

In spite of numerous chemical, physical and microscope studies of the pollen tube cell wall, the precise organisation and structure of the various components has been well characterized in many species such as Arabidopsis thaliana (Chebli et al. 2012), Lilium longiflorum (Lancelle and Hepler 1992; Jauh and Lord 1996), Nicotiana tabacum (Kroh and Knuiman 1982; Geitmann et al. 1995; Ferguson et al. 1998; Derksen et al. 2011), Petunia hybrida (Derksen et al. 1999), Pinus sylvestris (Derksen et al. 1999) and Solanum chacoense (Parre and Geitmann 2005) but are still a matter of conjecture. The tube wall is usually observed to comprise of two distinct layers, the outer layers begin fibrillar and the inner layer non-fibrillar and electron-lucent. According to Ferguson et al. (1998) the growing pollen tip is covered solely by the primary fibrillar wall. The inner layer, commonly called the callosic layer, is a secondary wall first visible some distance $(15 \mu \mathrm{m})$ behind the tip, and varies in thickness depending on age and physiological conditions.

In this context, to be precise, following Chebli et al. (2012), we quote: "Although the pollen tube does not form a conventional secondary cell wall layer, its wall is assembled in two phases. The "primary layer" is mainly formed of pectins and other matrix components secreted at the apical end of the cell. The "secondary layer" is assembled by the deposition of callose in more distal regions of the cell (Heslop-Harrison 1987). Depending on the species, cellulose microfibrils have been found to be associated either with the outer pectic or with the inner callosic layer. Unlike most other plant cells, cellulose is not very abundant representing only $10 \%$ of total neutral polysaccharides in Nicotiana alata pollen tubes, whereas callose accounts for more than $80 \%$ in this species (Schlüpmann et al. 1994)".

Using different methods of histochemical and specific labeling performed using antibodies, one can observe organization of the cytoarchitecture and relative spatial distribution of cell wall components in the pollen tube. The pollen tube cell wall consists of inter alia: pectins with low and high degree of esterification, callose, microfibrillar polysaccharides, crystalline cellulose, and fucosylated xyloglucans. About pectins location, we quote from Chebli et al. (2012): "Pectins with high degree of esterification were primarily found at the pollen tube tip, along the first $5 \mu \mathrm{m}$ of meridional length. The amount of highly esterified pectins decreased by two-thirds in the first 10 to $12 \mu \mathrm{m}$, where it reached a stable value that was maintained along the entire distal region. By contrast, the label for low esterified pectins was weak at the tip (around 10\% of the maximum label intensity determined in an individual cell) and increased 4-fold in the first 10 to $12 \mu \mathrm{m}$, where it reached a plateau".
Callose deposition began at a $10 \mu \mathrm{m}$ meridional distance from the tip and increased steadily until $40 \mu \mathrm{m}$, where it reached a plateau. It was shown that callose was deposited between the pectic fibrillar layer and the plasma membrane in the inner layer of the pollen tube cell wall. The Arabidopsis pollen tube cell wall comprised a fibrous component (probably cellulose microfibrils) whose main orientation was nearly parallel to the longitudinal axis of the cell (Chebli et al. 2012).

Concerning cellulose, Ferguson et al. (1998) states: "Cellulose co-locates with callose in the inner, electron-lucent layer of the pollen-tube wall, while both polymers are absent from the outer, fibrillar layer. Neither cellulose nor callose are present in the wall at the pollen-tube tip or in cytoplasmic vesicles. Cellulose is first detected approximately 5-15 $\mu \mathrm{m}$ behind the growing tube tip, just before a visible inner wall layer commences, whereas callose is first observed in the inner wall layer approximately $30 \mu \mathrm{m}$ behind the tip. Callose was present throughout transverse plugs, whereas cellulose was most abundant towards the outer regions of these plugs. This same distribution of cellulose and callose was also observed in pollen-tube walls of N. alata Link et Otto, Brassica campestris L. and Lilium longiflorum Thunb".

Xyloglucans inserted into the cell wall are associated to cellulose via hydrogen bonds, thus contributing to the formation of a tight network (Hayashi 1989; Acebes et al. 1993; Hayashi et al. 1994). Dardelle et al. (2010) suggested that fucosylated xyloglucans are cross linked to cellulose microfibrils because they were only associated with the inner layer of the Arabidopsis pollen tube wall. Furthermore, it was demonstrated that fucosylated xyloglucans were distributed evenly along the Arabidopsis pollen tube cell wall. It was suggested that a temporary failure to deliver these linker molecules to the cellular surface may lead to a transient widening of the tube diameter because of a lack of cellulose cross linking (Chebli et al. 2012).

While pollen tubes have a layered cell wall structure starting from the subapical region, containing a fibrilar pectic outer wall and an inner wall mostly composed of callose, the cell wall at the apex is almost exclusively composed of pectins. Neither cellulose nor callose are present in Nicotiana tabacum L. wall at the pollen-tube tip (Fergusson et al. 1998). A series of strategic enzymatic treatments also suggest that pectins, cellulose, and callose are highly cross-linked to each other (Chebli et al. 2012). The pollen tube cell wall, although enriched in pectins especially at the apical growing part, nevertheless consists of typical plant polymers (Winship et al. 2010). Therefore the proposed sliding of cell wall layers is restricted to take place in the subapex and regions even more distal to the pole, while the net effect should also be observed at the growing tube tip (wall thinning/thickening). For polar expansion dynamics and wall building we may also redirect the reader to 
a recent review article by Domozych et al. (2013), where the developmental dynamics of pollen tube growth, representing a spectacular example of anisotropic expansion in eukaryotes, is scrupulously studied.

It is clear that changes in turgor pressure may also influence pollen tube oscillations: A reduction in turgor pressure of only $0.02 \mathrm{MPa}$ can result in the immediate cessation of growth in the living cells (Taiz 1984; Messerli and Robinson 2003). This observation led to the loss of stability theory (Wei and Lintilhac 2003, 2007) stating that with an increase in turgor pressure the resulting wall stresses will gradually amplify to a definite critical value, at which time loss of stability must occur, leading to stress relaxation of the wall, wall material intercalation and consequent growth. Small pressure changes are also admitted by Winship et al. (2011): "Even in oscillating pollen tubes, small pressure changes can be expected, but they would be the result of wall relaxation-driven cell expansion, and not the cause". Bearing in mind the contributions of various wall building processes and exocytosis, we consider the behaviour of layered wall structures under changing turgor pressure regimes. For simplicity we restrict our discussion to the case of a quasi-homogeneous (layered) wall, and present a solution that demonstrates the importance of cell wall geometry.

\section{Material and Methods}

Nicotiana tabacum pollen was used for these studies. Anthers were harvested immediately before dehiscence and placed in desiccation chambers between 18 and $24 \mathrm{~h}$. Tobacco pollen was collected and stored at $-20^{\circ} \mathrm{C}$. Then, after removal, cells were held at room temperature for 25-30 min before suspending in standard germination medium [6\% (w/v) sucrose, $1.6 \mathrm{mM} \mathrm{H}_{3} \mathrm{BO}_{3}, 200 \mu \mathrm{M} \mathrm{CaCl}_{2}$ and $25 \mu \mathrm{M} \mathrm{MES} \mathrm{(pH}$ 5.5)]. Pollen was germinated and grown in culture chambers where it was assembled on microscope slides using silicone isolators for $3 \mathrm{~h}$ at $22.5^{\circ} \mathrm{C}$, before performing experiments on slabs containing $0.3 \%(\mathrm{w} / \mathrm{v})$ low gelling temperature agarose (plant cell culture grade, Type VII, Sigma) in $200 \mu \mathrm{l}$ germination medium.

Germinating pollen was used for imaging from $3 \mathrm{~h}$ to $5 \mathrm{~h}$ after the start of germination. Imaging was performed on a Zeiss Axiophot upright microscope, connected through a Nikon DXM/200 or UltraPix CCD camera which were run by NSI-Elements or UltraView morphometric software, respectively, to record information about the experiments (Centre for Advanced Microscopy, University of Amsterdam, Netherlands). The experimental method also involved recording fast time series images of the live pollen tubes. Microscope images were recorded (3 images per $1 \mathrm{~s}$ ) for $10 \mathrm{~min}$, and transferred to a computer for further analysis.
Mean resolution ratio from ten measurements equaled $0.02 \mu \mathrm{m} / \mathrm{px}$.

The width of cell wall was measured using the opensource morphometry software ImageJ in 3-s intervals, independently from the used camera. The width of the wall was measured by choosing a fixed point at the pollen tip, and then finding the perpendicular (to the long tube axis) distance to the wall at $1 \mathrm{R}, 2 \mathrm{R}, 3 \mathrm{R}$, etc., from the tip (fixed reference system, Fig. 4; here $\mathrm{R}=$ constant denotes the apical tube radius). These measurements gave a list of lengths (micrometre) that were imported into the Origin (Microcal) software. From length measurements, the wall width was calculated from the formula $l_{\text {outside }}(r)-l_{\text {inside }}(r)$, where $r=$ $1 \mathrm{R}, 2 \mathrm{R}, \ldots$ denotes the axial distance from the tip.

Matlab (The MathWorks ${ }^{\mathrm{TM}}$ ) code was used for model calculations.

\section{Results}

\section{Osmotic pressure dynamics in pollen tube growth}

The growth of the plant cell wall is determined by its viscoelastic, elastic, plastic, and shearing (rheological) responses to the mechanical stresses induced by turgor pressure. It has also been shown that the mechanical properties of the wall can be modified by the intercalation of specific new cell wall materials into the wall (Boyer 2009). In order to deconstruct the various wall behaviours we start with the Ortega (1985) equation:

$$
\frac{1}{V} \frac{d V(t)}{d t}=\Phi(P(t)-Y)+\frac{1}{\varepsilon} \frac{d P(t)}{d t}
$$

which formalizes the combined effect of inelastic $(\Phi)$ and elastic $(\varepsilon)$ components to the volume $V$ relative extension rate. It takes into account elastic deformation of the cell wall by introducing volumetric elastic modulus $\varepsilon$ for the changing turgor pressure $P(t)$. The coefficient $\Phi$ stands for the 'extensibility' of the wall which is defined as the reciprocal of the materials' viscosity (Dumais et al. 2006). Equation (1) describes the relative rate of change in volume of the cell chamber, as the sum of irreversible and reversible deformation of the wall. In Eq. (1) $P(t)$ stands for the dynamic turgor pressure inside the vacuole (as a function of time $t$ ), while the constant $Y$ describes a yield threshold value, the value that must be exceeded for growth to occur. The relative change in volume can be calculated when the turgor pressure $P(t)$ dependence on time is known.

The goal of the present analysis is to derive an alternative model that relates changes in osmotic pressure to the observed growth dynamics (Hill et al. 2012). Considering the volume of the elongating pollen tube let us partition the total cell volume $V_{\mathrm{T}}$ into two compartments $V_{\mathrm{A}}$ and $V_{\mathrm{B}}$ where 
$V_{\mathrm{B}}$ is the volume of the cylindrical pollen tube basal to the apex and $V_{\mathrm{A}}$ is the volume of the apical region itself, that is known to undergo volume oscillations (Zonia et al. 2006). $V_{\mathrm{T}}(t)=V_{\mathrm{B}}(t)+V_{\mathrm{A}}(t)$ is a function of time $t$. Experimental data suggests that there is a continuous transition in the mechanical properties of the cell wall between the apical and basal regions (Geitmann and Parre 2004). We further assume that $V_{\mathrm{B}}(t)=u_{0} t$ and $\left[u_{0}\right]=\mu \mathrm{m}^{3} / \mathrm{s}$ (the rate of the volume increase), where $V_{\mathrm{B}}$ is considered for the quasi-linear phase of elongation growth, where this approximation holds.

To describe apical volume oscillations in the pollen tube we utilize Eq. (1) in the modified form, where time dependence of $P$ is given by a pressure fluctuation term (first correction) $\delta P(t)$, so $P(t)=P+\delta P(t)$. Therefore Eq. (1) takes the form

$$
\frac{1}{V_{A}} \frac{d V_{A}}{d t}=\Phi(t)(P+\delta P(t)-Y)+\frac{1}{\varepsilon} \frac{d(P+\delta P(t)-Y)}{d t}
$$

This first-order differential equation takes into account elastic $(\varepsilon)$ deformation of the cell wall. The constant value of $\varepsilon$ simplifies the mathematical analysis of Eq. (2). In this we couple the Ortega equation to the equation for the change in cell wall properties by taking the extensibility coefficient $\Phi$ that is responsible for plastic wall properties as a dynamic variable. We assume that the intracellular osmotic pressure can be changed in response to changes in the extracellular osmotic potential, therefore the osmotic pressure term, which is included in $\delta P(t)$, must be also introduced. Hence, Eq. (2) becomes:

$$
\frac{1}{V_{A}} \frac{d V_{A}}{d t}=\Phi(t)(P+\delta P(t)-Y)+\frac{1}{\varepsilon} \frac{d(\delta P(t))}{d t}
$$

Because the $Y$ time-derivative vanishes, Eq. (3) shows that the relative change $V_{\mathrm{A}}{ }^{\prime} / V_{\mathrm{A}}$ of the apical volume $V_{A}$ is dependent on the sum of the plastic properties term proportional to the total turgor pressure $P+\delta P(t)-Y$, plus the elastic properties term (in the range of applicability of the Hooke's law) conjugated to the pressure fluctuations derivative $\delta P(t)$. This relationship may be associated to the phase shift that has been frequently observed and discussed (McKenna et al. 2009). We note that the material constants responsible for the properties of the growing wall are included in $\Phi$ and $\varepsilon$, combining irreversible growth and reversible cell extension, respectively. By taking $\Phi=\Phi(t)$ and the constant $\varepsilon$ (in the apex), we may follow Pietruszka (2010, 2012) to introduce dynamic cell wall mechanical properties by the inclusion of the wall-loosening factor chemical activity. Cell wall visco-elasticity is expressed by $\Phi(t)=\Phi_{0} x_{0} \exp \left(-k_{0} t\right)$ term, where $\Phi_{0}=$ constant is the extensibility coefficient proposed originally by Lockhart (1965) with the dimensionality $\left[\Phi_{0}\right]=10^{-6} /(\mathrm{s} \cdot \mathrm{MPa})$, which must be retained. The terms $x_{0}$ and $k_{0}[1 / \mathrm{s}]$ represent initial polymer density $\left[x_{0}=x\left(t=t_{0}\right)\right]$ and cell wall intercalation rate, respectively. The latter needs a comment. The equations used in the modelling are based upon a paper (Pietruszka 2010) interpreting behaviour of plant cell walls in terms of rapidly decaying expansin activities, though mathematically equivalent more parsimonious explanations are available (exponential decay of creep rates and in stress relaxation).

By substituting the explicit form of $\Phi(t)$ in Eq. (3) we obtain:

$$
\frac{1}{V_{A}} \frac{d V_{A}}{d t}=\Phi_{0} x_{0}(P+\delta P(t)-Y) e^{-k_{0} t}+\frac{1}{\varepsilon} \frac{d(\delta P(t))}{d t}
$$

Eq. (4) represents a hydrodynamic equation that takes into account the effective pressure in the system and also (in the first approximation) the cell wall elastic and plastic properties. This equation introduces deposition of new cell wall material into the existing cell wall at a rate $k_{0}$, with the initial concentration of pectins that are used for new cell wall growth denoted as $x_{0}$. The constant turgor pressure $P$ stands for the 'first fluid'. The pressure fluctuation term ('second fluid') can be given by $\delta P(t)=R T\left(\Delta C+\sigma_{S} \Delta C_{S}\right)$ where $C$ denotes the concentration of non-permeable solutes, $C_{S}$ denotes the concentration of permeable solutes, and $\sigma_{\mathrm{S}}$ is the reflection coefficient for permeable solutes. The volume flow at a given temperature $T$ has two components, the first being a hydraulic driving force represented by the hydrostatic (or turgor) pressure $P$, and an osmotic driving force represented by the osmotic pressure gradient $\delta P(t)$. Thus, after an extracellular osmotic pressure decrease, an influx of permeable solutes, or a change in the intracellular solute concentration due to metabolism, water influx will occur.

Conceptually we break down the oscillating turgor phenomenon into two components, as if it were made up of one constant source of fluid pressure superimposed on which is a second variable pressure treated as if it were contributed by a second fluid. This reflects the assumption that these two sources of pressure may arise from different system dynamics. By analogy one might consider the experience of being in a concert hall, where a variable tone originating from one source is superimposed on a pure tone originating from another, creating a changing landscape of beat frequencies. This mathematical device is permitted because pressure is subject to the superposition principle. In fact it is important to understand the nature of the two fluids in order to understand their Fourier contributions.

In reality however, the formal differentiation of the oscillating pressure into two fluids is not free from mathematical consequences.

After integrating Eq. (4) with respect to time, the solution takes the logarithmic form

$$
\begin{aligned}
\log \left(\frac{V_{A}}{V_{0}}\right)= & \Phi_{0} x_{0} \int_{t_{0}}^{t} e^{-k_{0} \tau}[P+\delta P(\tau)-Y] d \tau+ \\
& +\frac{1}{\varepsilon}\left[\delta P(t)-\delta P\left(t_{0}\right)\right]
\end{aligned}
$$


where $V_{\mathrm{A}}=V_{\mathrm{A}}(t)$, and $V_{0}=V_{\mathrm{A}}\left(t_{0}\right)$. We notice in Eq. (5) that the expanding pollen tube tip volume $V_{\mathrm{A}}(t)$ cannot be integrated without knowing the explicit form of the effective pressure $P+\delta P(t)-Y$ time dependence. Eq. (5) can serve for calculation of apical volume changes due to osmotic pressure fluctuations. However, because pollen tube growth will be described by its total volume $V_{\mathrm{T}}(t)=V_{\mathrm{B}}(t)+V_{\mathrm{A}}(t)$, growth rate will be approximated by using the time derivative: $V_{\mathrm{T}}^{\prime}(t)$ $=V_{\mathrm{B}}{ }_{\mathrm{B}}(t)+V_{\mathrm{A}}^{\prime}(t)$, providing that the continuity condition for the volume flow is fulfilled. The logarithmic relationship in Eq. (5) means that even small fluctuations of the effective pressure, for example those caused by the osmotic pressure gradient generated by water movement across the plasma membrane, may lead to relatively large amplitude fluctuations in apical volume. Further amplification comes from the fact that osmotic influx occurs over the entire length of the pollen tube (which would also amplify slight changes in osmotic water uptake at the tip) whereas the resulting growth is all focused in the tip.

\section{Fourier decomposition}

Although many aspects of plant growth can be described in terms of first order system dynamics (Erickson 1959, 1986), not all aspects of plant growth dynamics can be adequately described in such simple terms. Because pollen tube growth presents us with an easily observed example of time variable growth behaviour in a single cell we are afforded an unique opportunity to explore the dynamics of more complex cell wall behaviour through harmonic analysis. To this end we have undertaken the Fourier decomposition of Eq. (4). After taking the Fourier transform (Harris 1998) of Eq. (4) the left side becomes the Fourier transform of the relative growth rate $F(\mathrm{GR})$, and the right side can be split into 3 contributing terms, which we may call plastic, fluctuating-plastic and fluctuating-elastic, respectively, and the corresponding energy terms are proportional to:

$$
\begin{aligned}
& \frac{1}{k_{0}^{2}+4 \pi^{2} f^{2}} \\
& \left|F(\delta P(f)) * \frac{1}{k_{0}+2 \pi i f}\right|^{2} \\
& f^{2} \mid F(\delta P(f))^{2}
\end{aligned}
$$

Here $f$ stands for oscillation frequency (in $\mathrm{Hz}$ ), ${ }^{*}$ denotes convolution, and $F$ denotes the Fourier transform. Terms (6)-(8) stand for all model contributions to the energy distribution spectrum (see Fig. 1 obtained in Matlab code). The first, plastic component, expression (6) has its maximum at $f=0$ and a Lorentz form for higher frequencies. This term is very unlikely to contribute to oscillations, but it can be responsible for steady elongation. The appearance of any oscillations $(f>0)$ will compete for a spectral density allocated to this term. The third term (8), fluctuating - elastic, will have an envelope of $f^{2} F(\delta P)$ (inset in Fig. 1). This will necessarily be 0 at $f=0$ and will increase with frequency. This term will naturally promote the oscillatory state. The second component (7), fluctuating - plastic, is a non-trivial one and will act as an effective coupling between the first and last terms. The interplay between these terms will define the effective resonant frequency of oscillations in the pollen tube (Haduch-Sendecka et al. 2014).

\section{A simple shear model of pressure-induced cell wall thick- ness variations}

The dynamics of the growth process and the final cell shape and size result from the mechanical features of the cell wall

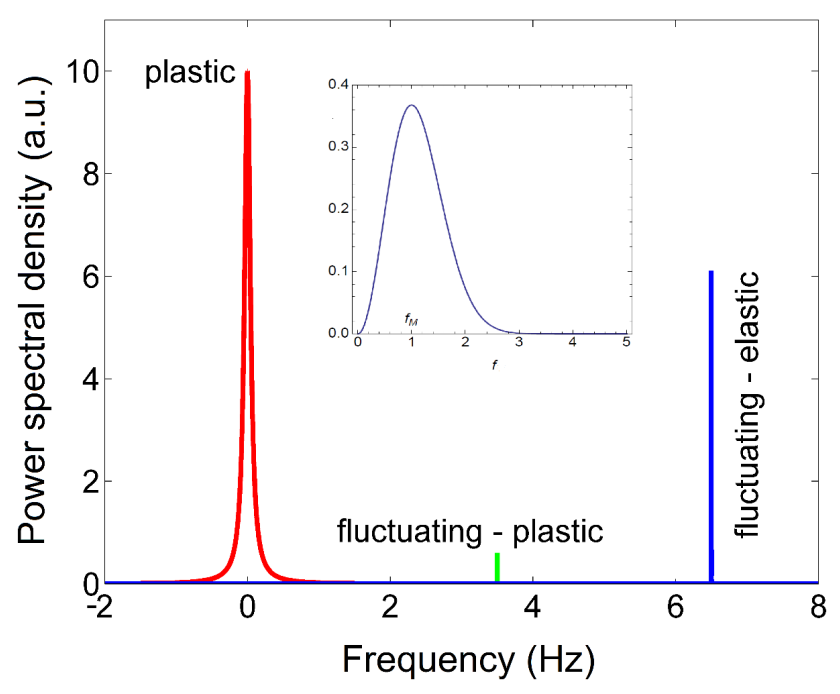

Figure 1. Power spectral density as a function of frequency of a growing pollen tube (model calculations). The plastic, fluctuating plastic and fluctuating - elastic contributions to energy are calculated from mathematical expressions (6)-(8), respectively. The plastic term given by expression (6), responsible for steady elongation, has a maximum at $f=0$ and a Lorentz form for higher frequencies. The fluctuating - plastic term (7) will act as an effective coupling between the first and last terms (6) and (8), see also Fig. 4. The third term (here in the Dirac delta form), expression (8), fluctuating - elastic in origin will naturally promote the oscillatory state. A clear 'resonant' value for frequency will most likely be established by the coupling term and external constraint (osmotic concentration). The elastic part is shown to be capable of producing a stationary solution with the envelope $f^{2} \exp \left(-f^{2}\right)$ with a non-zero resonant frequency $f_{\mathrm{M}}$ even from purely Gaussian fluctuations (inset). It was found to compete for spectral density with the plastic 'steady growth $f=0$ ' component responsible for the elongation. 'Tug-of-war' is done though the terms encapsulated in expression (7). Simulation parameters (arbitrary): $k_{0}=0.1 \mathrm{~s}^{-1}, \omega=11 \mathrm{rad} / \mathrm{s}, \varphi=0 \mathrm{rad}$. 
and turgor pressure. Any mechanistic framework of plant growth must therefore include the layered construction of the wall. Our model assumes a quasi-multi-layered wall structure where the individual layers consist of cellulose microfibrils embedded in a hemicellulose matrix with linkers, such that each layer can be treated as a discrete network constrained by inter-layer hemicelluloses bridges that serve to control the sliding of the layers. Further information about the ramifications of the micro/nano-scale geometry of the cell wall on the mechanics of cell wall thickness fluctuations observed in pollen tubes can also be useful even minor changes at the micro/nanoscale can have major downstream consequences in terms of the temporal behaviour of the cells (see Fig. 2b in Chavarria-Krauser and Yejie 2011 - note the 'simple sheared' bricks). Here, we propose a simple model where we suggest that these wall thickness

A

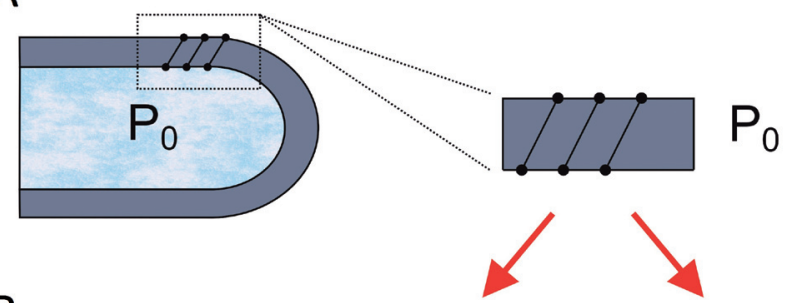

B
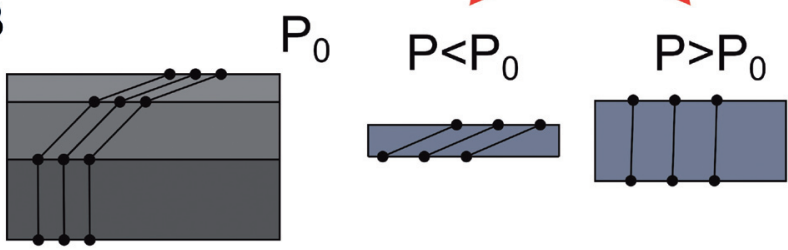

C

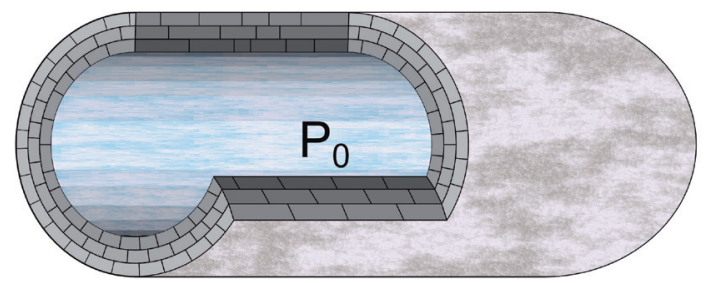

Figure 2. A model that portrays that cell wall layered structure may allow pressure-induced changes of wall thickness in the apical part of pollen tube (anomalous Poisson ratio case): $P$ - turgor pressure, $P_{0}$ threshold pressure (not to be confused with $Y$ - the yield threshold). Wall thickness influenced by the oscillating turgor pressure: A. Single layer case. B. Multi-layer case - the cell wall thickness fluctuations due to the changes in turgor pressure and cell geometry may also take place for a positive value of Poisson ratio (see the text). C. Concentric single layers represented by uniaxial cylindrical tubes at the apical end of pollen tube (right). The axially sliding layers due to pressure $\delta P(t)$ variations highlighted by the shaded area of the cross-section. Compare with Fig. 2 in Geitmann and Ortega (2009). oscillations are due to this specific wall structure acting in concert with effective pressure fluctuations, wall building and exocytosis.

To keep order we consider negative (anomalous) and positive (normal) Poisson ratio in taking into account layers and link geometry.

\section{Anomalous Poisson ratio}

In this model we assume the inter-layer linkers consist of stiff polymer bonds of almost equal length connecting the neighbouring wall layers (see Figs. 2-3 for illustration). With a value of turgor pressure $P$ equal to a certain constant threshold value $P_{0}$ the cell wall possesses a definite thickness $d_{0}$. However, due to the total turgor pressure oscillations (as caused by $\delta P(t)$ in our model Eq. (4)) $P$ can be either greater or smaller than this constant value $P_{0}$. Consequently, the periodic changes in turgor pressure would compress the hemicellulose inter-layers thereby rotating the angle of the

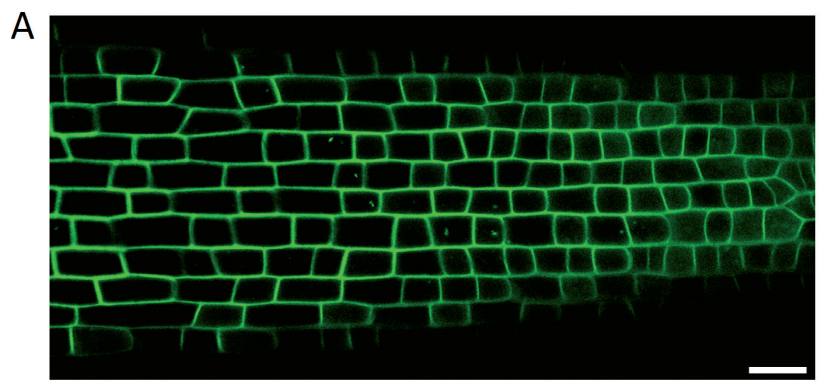

B

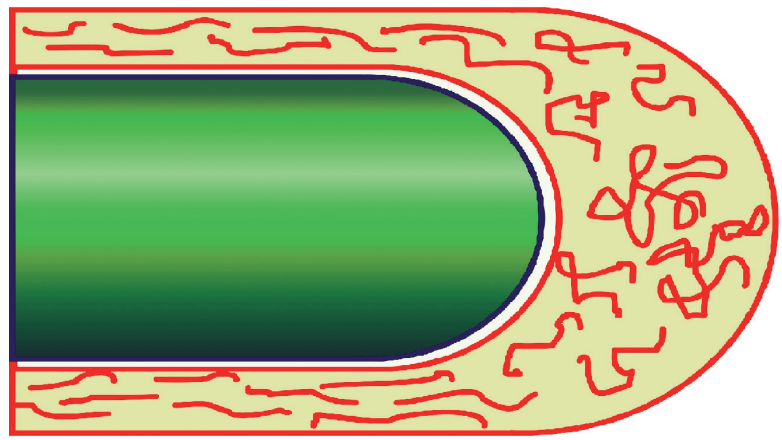

\section{LEGEND}

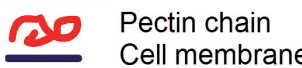

Figure 3. Cell pattern of epidermis in Hordeum vulgare L. roots of 'Karat' cultivar (wild-type plant). A. Autofluorescence of the cell wall was analysed by CLSM (confocal laser scanning microscopy) according to procedure described by Marzec et al. (2013). Scale bar $=20 \mu \mathrm{m}$. B. A schematic view of a pollen tube tip as considered in this paper (see the text). Pectin chains and cell membrane indicated. Upper chart courtesy of Marek Marzec, Deptartment of Genetics, Faculty of Biology and Environment Protection, University of Silesia, Katowice, Poland. 
hemicellulose polymer bonds linking the cellulose layers and enabling limited slippage between the layers and oscillations in the cell wall thickness (Fig. 2). However, this model, slightly modified, still holds for a more realistic multi-layered system constituting the cell wall.

Imagine that the layers are stacked one upon another. Then due to the increased inner pressure $P>P_{0}$ the sliding of the first layer will result in the re-positioning of the bonds between the first and the second layers more normally (perpendicular to the plane of the layers, Fig. 2). This will, however, cause increased pressure on the second layer (we count from the inner surface of the cell) causing it to slide and re-positioning the polymer bonds to some arbitrary angle. This process (inter-layer bond rotation) travels through the wall thickness in a radial direction as a bond polarization wave until it reaches the outer-most layer. Thanks to this we obtain the multi-layer (composing the cell wall) thickening leading to the maximum wall thickness equal (in a rough approximation) $(n-1) l \tan (\alpha)$ times the width of a single layer, where $n$ - is the number of layers, $l$ - overall bonds length and $\alpha$ - the slope angle of a single bond. The opposite process will take place if $P<P_{0}$ when shrinking of the wall occurs. This mechanism may be responsible for the observed cell wall thickness fluctuations (Kroeger et al. 2011, Fig. 2B) which we propose to be caused by slightly oscillating effective pressure and special geometry of the pollen tube apical region. Further, by assuming water incompressibility and Pascal's principle, the expansion and contraction of the apical zone of a pollen tube wall may be induced by the effective pressure changes in the vacuole.

\section{Normal Poisson ratio}

If the hemi-cellulose bonds are not stiff, as assumed above, but compliant (in this study we cover both possibilities), an alternate scenario can be developed: a multilayer sliding may arise due to the hemispherical geometry of the apical part and axially-exerted compressive forces, inducing contraction under pressure and expansion of the wall thickness caused by inter-layer hemicellulose/pectin matrix when inner pressure is released (wall building processes may occur). Nevertheless, similar conclusions as above can be drawn: the pressure changes in the vacuole by performing subsequent longitudinal sliding of the wall layers progressing from the inner to the outer surface of the wall, in another form of a 'polymer bonds polarization wave', may cause observable changes in the wall thickness in the apical zone. Apparently, a similar mechanism may be responsible for the analogous effect at the shank zone, however in much lesser degree because of stiffening of the system due to maturation processes - strain hardening (the walls become inextensible and more resistant to the internal pressure changes). The authors realize that the conventional explanation of the experimentally observed oscillations in cell wall thickness (McKenna et al. 2009), i.e. oscillating exocytosis rate, is widely accepted. Nevertheless, our explanation through 'polymer bonds polarization wave' may be treated as supplementary alternative. The ageing process can be described by a constant rate Poissonian decay process as recently proposed in Eggen et al. (2011) leading to the notion of a gradient in material properties over the apical region and attenuation of above described processes. This is probably the case which is observed in experiment (Winship et al. 2010, Fig. 2b-d): apical cellular softness vs. basal cellular stiffness (and our own experimental results presented in Fig. 4), from which we can conclude that wall thickness fluctuations are larger in the apical (subapical) than in the basal region.

The proposals presented above can be described mathematically (Atkin and Fox 2005) as a kind of simple shear deformation (which is a special case of deformation in which only one component of velocity vectors has a non-zero value): $\boldsymbol{x}=G(\boldsymbol{X}, t)=\boldsymbol{X}+2 a(t) X_{2} \boldsymbol{e}_{1}$ where $G$ maps each point $\boldsymbol{X}$ of the reference configuration onto its position $\boldsymbol{x}$ at time

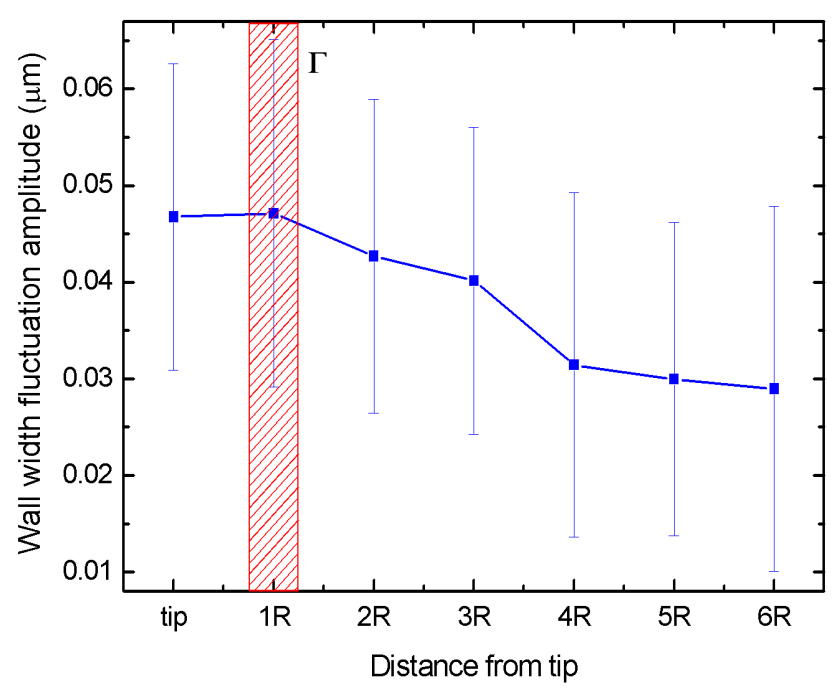

Figure 4. Variation in the magnitude of changes in wall thickness for Nicotiana tobacum pollen tubes as a function of the distance from the tip measured in terms of the tube radius $\mathrm{R}$ ( $1 \mathrm{R}$ equals here $4.14 \mu \mathrm{m}$ ). Data points (dots) are taken from our own experiment (AH-S), where the number of samples $n=37$ (resolution $\sim 0.01 \mu \mathrm{m}$ ). Error bars indicate standard deviation. The maximum of the plot at a distance $1 \mathrm{R}$ from the tip stays in agreement not only with the predictions of the frustration model (Pietruszka 2013, $\Gamma$ - interface in Fig. 1A) and self-regulating role of cell wall ageing presented in Fig. 6 by Eggen et al. (2011), but also with the cellular stiffness data, acquired with a microindenter, plotted against the distance from the pole measured on the central longitudinal axis of Arabidopsis pollen tube (see a local minimum at $\sim 5 \mu \mathrm{m} \approx 1 \mathrm{R}$ in Chebli et al. 2012, Fig. 9, naturally corresponding to the maximum in the wall width fluctuation amplitude at $1 \mathrm{R}$ in our case). 
$t$. The latter term must be defined in concert with the value of the observed thickening/shrinking of the apical wall. The simple shear already gives the effect of layer slippage (Constantinescu and Korsunsky 2007), which can be imagined as the top of a stack of playing cards being pushed to one side, making cards glide on top of each other. The calculated rotation tensor corresponds to rotation by the angle $\theta(t)=-$ atan $a(t)$ or in classical notation $a(t)=\tan (\alpha t)$. So the multilayered wall material deformation takes the form $\boldsymbol{x}=G(\boldsymbol{X}, t)=\boldsymbol{X}+2$ $X_{2} \tan (\alpha t) \boldsymbol{e}_{1}$ and as it should be expected - depends on time. Such deformation may be linked with the dynamic pressure fluctuations in the apical region, especially when coinciding with quantized exocytosis with the change of interlayer distance. Simply put, the fluctuations of the turgor pressure in the apical region, which affect the innermost layer of the cell wall, cause the increase/decrease of the cell wall thickness in the neighbouring region. The layers are bound in different ways in the apical and the basal parts of the structure, what is the consequence of anisotropy (Baskin 2005) of cell wall structure (see Fig. 3, the symbolically illustrated 'red' pectin chains). Because the layers are connected, the innermost layer, which is in stress, drags the next layer, etc. The 'drag effect' is manifested on bigger scale in the anisotropic structure and can cause wall shrinking (similarly as when shearing a rectangle we obtain a parallelogram whose height is smaller than the rectangle). The outlined scenario may possibly report on cell wall thickness changes in the apical region in pollen tubes but also, in concert with cylindrical (shank) plus hemispherical (apex) geometries, may form an auxiliary model for the rapid polarized growth of pollen tubes.

\section{Discussion}

Our proposal, despite inevitable degree of simplification, is in accord with the new physics of cell wall behaviour expressed by the loss of stability model (Wei and Lintilhac 2007) and cell wall thickness oscillations, detailed in Kroeger et al. (2011). In fact, the phase data provided by Kroeger et al. (2011) and by McKenna et al. (2009) ought to offer an obvious answer as to whether the Poisson's ratio is positive or negative, but these are not consistent with one another. Loss of stability is a result of gradual loading (internal pressure) process: "With a gradual increase in internal pressure the resulting stresses in the wall will gradually increase to a critical value, at which time loss of stability must occur, leading to stress relaxation in the wall" (Panovko and Gubanova 1965; Wei and Lintilhac 2007). This loss of stability takes place due to small fluctuations in turgor pressure (neglected in first approximation in Pietruszka 2013) and wall building (in each cycle), and results in rapid pollen tube elongation. The present model is not only in agreement with the recent investigations connected with expansion propensity as a function of the distance from the apex (see Fig. 2 in Eggen et al. 2011), but also with the cellular stiffness data (see Fig. 9 in Chebli et al. 2012), compare with Fig. 4 in this study. Our analysis based on sliding wall layers allows for curvature driven delivery (exocytosis) close to the curved part where the apex joins the subapex in the pollen (Zonia and Munnik 2008). The mechanism of sliding wall layers will also lead to the cumulated apical tip wall thickening (see Figs. 1 and 4 in McKenna et al. 2009). It has also been shown (Koch 1982, cited after Eggen et al. 2011) that the local state of the wall controls the local rate of incorporation of new material and that this is a feedback-controlled mechanism; however, no feedback mechanism was indicated. In our proposal a feedback mechanism is represented by small fluctuations, with the amplitude of about $0.01 \mathrm{MPa}$ (the measurements of Benkert et al. 1997, reanalysed in Fig. 3c by Zonia and Munnik 2011, which had a resolution of $0.005 \mathrm{MPa}$, and thus possessed sufficient sensitivity to see the changes of the order of $0.01 \mathrm{MPa}$ ), also in agreement with 'loss of stability model' numerical value of $0.02 \mathrm{MPa}$, strong enough - due to logarithmic relation between pressure and volume - and enhanced by the fact that osmotic influx occurs over the entire length of the pollen tube, Eq. (5), to provide such mechanism through wall layers sliding.

The latter needs a comment. Seemingly, there are very good reasons for why the turgor pressure does not fluctuate. The first reason is that there is no water pump: the uptake of water necessary to generate turgor pressure derives from a change in the water potential of the cell. The second reason is connected with the hydraulic conductivity of the plasma membrane. It is commonly accepted that the plasma membrane of plant cells in general, and pollen tubes in particular is very leaky; should any increase in turgor pressure occur, this will be 'immediately' offset by the expulsion of water, with the resulting return to the original pressure. However, because no 'immediate' changes may occur, this is what can be considered as high frequency, low amplitude pressure fluctuations. Since 1965 there have been countless examples of experiments in which the parameters controlling growth have been tested. These studies show that growth is due to underlying changes in the cell wall, and not to changes in turgor pressure. A very concise summary on this subject can be found in the textbook, Plant Physiology by Taiz and Zeiger ( $4^{\text {th }}$ edition, 2006). Nonetheless, the paradigm referring to the chemical wall loosening (cell wall model) should at least admit stepwise decrease of turgor pressure, clearly visible in a landmark study by Benkert et al. (1997) and further reanalyzed by Zonia and Munnik (2011). Nonetheless, such decrease should be next accompanied by pressure recovery (presumably due to wall layers slide in the relaxation individual step) forming a distribution of fluctuations about pressure equilibrium value $0.2 \mathrm{MPa}$ (Fig. $3 \mathrm{c}$ in Zonia and Munnik 2011). There, the authors claim that the 
measured periodicity for pressure oscillations ranges from $12 \mathrm{~s}$ to $25 \mathrm{~s}$, which is the same as routinely reported for oscillatory dynamics in lily pollen tubes (Mc Kenna et al. 2009; Zerzour et al. 2009). The situation is, however, even more appealing: by retrieving the fluctuating pressure values from the chart (Fig. 1a in Winship et al. 2010, the monotonically descending linear part), a single frequency peak in the pressure power spectrum has been detected by discrete Fourier analysis (FFT), see Fig. 5 and the caption to this figure. Thus, by using FFT and the Lorentzian fit we showed, that turgor pressure in short instants increases in a strict periodical manner, presumably in advance in the increase in growth (or increase in wall thickness). The latter fact, however, may invoke a significant paradigm shift.

We formulated a simple mechanism consistent with the recent results (Rojas et al. 2011), where chemically-mediated deposition causes turnover of cell wall cross-links, thereby facilitating mechanical deformation. This mechanism can also reflect the pectate structure and suggested distortion by Boyer (compare Boyer 2009, Fig. 5 c,d) in Chara cell walls under tension as originating from $P+\delta P(t)$. It is concluded (Boyer 2009) that growth (in Chara) is controlled by a nonenzymatic reaction in the wall itself. Among others, this reaction depends on pressure $P$ - when turgor pressure rises in cells (by the amount of $\delta P(t)$ ), it puts wall polymers in tension and make the load bearing bonds susceptible to calcium loss and allow polymer slippage that irreversibly deform the wall. In accord with our present proposal, Proseus and Boyer (2007) suggested that the ladder like structure would be susceptible to distortion by $P$ : at low $P$ (corresponding to $P-\delta P(t)$ ) the ladder would straighten (Fig. 5C in Boyer 2009 corresponding to Fig. 2B in this paper). Then, above a critical $P\left(P_{\text {crit }} \sim P+\delta P(t)\right)$, distortion would increase the distance between adjacent galacturonic residues (Fig. 5D in Boyer 2009). When $\mathrm{Ca}^{2+}$ cross-linked pectates are placed under physical tension, as imposed by turgor pressure $\sim 0.2$ $\mathrm{MPa}$, the bonds may lengthen and thus weaken and decrease their affinity for $\mathrm{Ca}^{2+}$ (Proseus and Boyer 2007; Boyer 2009). Dissociation may occur, allowing turgor dependent expansion (see also Fig. 4 in Pietruszka 2013).

Apparently, our model proposes that small oscillations of turgor are the causative agent for oscillations in wall thickness. This is the converse of the proposal by Winship et al. (2010) which postulates that variations in cell wall mechanical properties cause the (growth rate) oscillation and that variations in turgor (if there are any) are a passive consequence due to cell wall relaxation. Whereas the LOS model, as it is used by Zonia et al. (2006), proposes gradual increase in turgor until a threshold when rupture of individual links between cell wall polymers occurs, Winship et al. (2010) state that turgor is essentially stable, but that an exocytosisinduced relaxation of the wall causes expansion. The present paper does not arbitrate which explanation is the proper one.

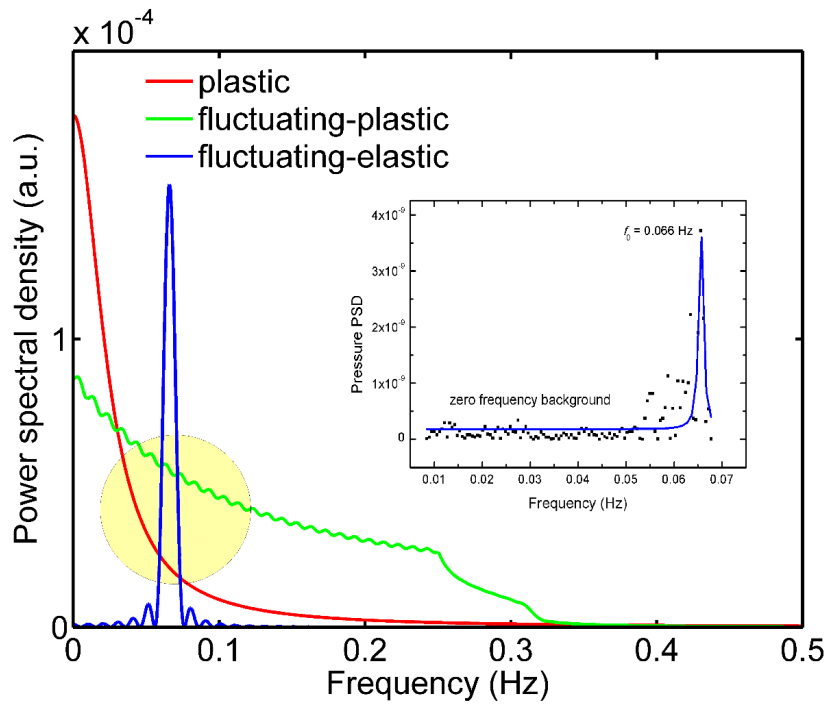

Figure 5. Theory: The energy spectrum obtained in model calculations, to bridge the gap between the modelling and the behaviour of pollen tubes in vivo. The circle centred at the frequency $f_{0}$ $\approx 0.066 \mathrm{~Hz}$ (period $T_{0} \approx 15.24 \mathrm{~s}$ ), shows the area with all contributing terms (6)-(8) present, allowing for simultaneous periodical motion and visco-elastic (plastic) cell extension. Experiment: The inset presents the turgor pressure power spectrum (Power Spectral Density - PSD) obtained for the data presented in Fig. 1a by Winship et al. (2010) and reanalyzed in Fig. 3 b by Zonia and Munnik (2011). A single non-zero frequency $f_{0}$ obtained by the detrended (baseline subtracted) Fourier analysis (solid dots) is indicated in the chart. The Lorentz resonance curve fit is denoted by solid line $\left(f_{0}=0.06562 \pm 0.00006 \mathrm{~Hz}\right.$, half-width $w=0.00107 \pm 0.00013$ $\left.\mathrm{Hz} ; \chi^{2}=8.9224 \times 10^{-20}\right)$. According to the presented model the "resonant" value for frequency will most likely be decided by the coupling term. The obtained single valued frequency $f_{0}$ reveals strict periodicity in a form of regular changes in turgor pressure of growing Lilium longiflorum (lily) pollen tubes, originally measured in pressure probe experiment by Benkert et al. (1997), which may be attributed to the value of maximum $\mathrm{Ca}^{2+}$ influx in the cell wall apex for lily (Fig. 3 in Holdaway-Clarke and Hepler 2003). Since basically a prominent single peak in the spectrum appears (inset), no white (Gauss) noise may be the cause of oscillations. The experimentally obtained Lorentz peak is corresponding to the Lorentzian centred at $f_{0}=0.066 \mathrm{~Hz}$ (model calculations). Simulation parameters read: $k_{0}=0.015 \mathrm{~s}^{-1}, \varphi=\pi / 2$, amplitude $p_{0}=0.01 \mathrm{MPa}$.

In our opinion the fluctuations $\delta P(t)$ may serve as recovery pressure after each growth cycle and may be either a cause or an effect in accord with expressions (6) - (8). In fact, charge separation due to pressure-induced wall distortion (dislocations), as a mechanism for restoring equilibrium in this non-equilibrium (growing) system of sliding (multiple) layers, is implicitly proposed in this study. The responsible force $\boldsymbol{F}=\mathrm{q} \boldsymbol{E}=-\operatorname{grad}(V)$, where $\boldsymbol{E}$ is the electric field vector, q the effective charge and $V$ is the potential difference, may 
eventually lead to the observed $\pm 0.01 \mathrm{MPa}$ pressure fluctuations (see also Fig. 5 in Barbacci et al. 2013 presenting a $0.54-0.52=0.02 \mathrm{MPa}$ pressure drop and recovery fitted to Proseus and Boyer (2008) experiments).

In conclusion, whether we deal with positive or negative Poisson ratio, the 'multi-layered composition' of cell wall connected by hemicelluloses will inevitably produce cell wall thickness fluctuations under changing turgor pressure. These thickness changes occur mainly in the pollen tube apical region, where they are especially easy to follow. However, the hemispherical geometry (see Fig. 4 in Eggen et al. 2011) of this region may also play an important role, due to different force (per steradian) exerted onto the pollen tube tip as compared to the force exerted on the walls in the shank region. In this context the very fast process of elongation may be connected with oscillations of the additional space (volume) between the apical and subapical layers thus enhancing wall building and exocytosis.

\section{Conclusions}

We presented a model for pollen tube growth that is founded on the concept that oscillations in cell wall thickness are due to complex, turgor pressure induced distortion dynamics caused by the sliding of neighbouring layers of the cell wall.

Just as we learn about the composition of an acoustical or optical landscape from its Fourier components, so we may be able to learn about the mechanical landscape of pulsating growth of the pollen tube from its Fourier components. Potentially Fourier analysis may be able to distinguish between various causalities. Is the pulsating growth we see in pollen tubes caused by pulsating pressure or is it caused by transient changes in wall composition? Alternatively we might postulate that it is caused by neither, and that it is primarily due to mathematically demonstrable instabilities that depend on a suite of interacting behaviours.

Multilayered pollen tube walls can be modelled as consisting of multiple concentric coaxial layers (coaxial cylindrical tubes) which are coupled with one another via the inter-tube hemicelluloses. Distinct end constraints (apical $v s$. basal end), treated as 'boundary conditions', imposed on constituent cylindrical layers undergone to pressure variations (of the order of $0.01 \mathrm{MPa}$ compared to $0.2 \mathrm{MPa}$ turgor pressure $(\sim 10 \%)$ for lily pollen tubes (Zonia and Munnik 2011)) may lead to measurable oscillating behaviours (or statistical fluctuations) along the cell. As it was pointed out earlier, these heterogeneous space boundaries (at both ends) and the movement of individual tubes may influence the dynamic stiffness along the pollen tube wall. The fluctuations in turgor pressure causing propagation of the movement of concentric individual tubes should lead to alteration of the fundamental (isotonic) vibration mode. It seems, that the dynamic stiffness of the whole coupled system (uniaxially coupled tubes) influenced by turgor pressure plus changing osmotic pressure may be responsible for the observable wall thinning/thickening giving space for step-wise (quantized as opposed to continuous) wall building and exocytosis at the apical end.

Continuous transition from highly nonlinear (periodic) growth, to monotonically ascending and asymptotically saturated (sigmoid-like) growth, is predicted in the framework of the presented formalism.

Acknowledgment. We thank Dr. Laura Zonia and the Swammerdam Institute of Life Sciences, University of Amsterdam, Netherlands, where the measurements were performed (AH-S).

\section{References}

Acebes J. L., Lorences E. P., Revilla G., Zarra I. (1993): Pine xyloglucan. Occurrence, localization and interaction with cellulose. Physiol. Plant. 89, 417-422 http://dx.doi.org/10.1111/j.1399-3054.1993.tb05192.x

Atkin R. J., Fox N. (2005): An introduction to the theory of elasticity. Dover Pub, Mineloa, New York

Barbacci A., Lahaye M., Magnenet V. (2013): Another brick in the cell wall: Biosynthesis dependent growth model. PLoS ONE 8, e74400 http://dx.doi.org/10.1371/journal.pone.0074400

Baskin T. I. (2005): Anisotropic expansion of the plant cell wall. Annu. Rev. Cell Dev. Biol. 21, 203-22 http://dx.doi.org/10.1146/annurev.cellbio.20.082503.103053

Benkert R., Obermeyer G., Benturp F.-W. (1997): The turgor pressure of growing lily pollen tubes. Protoplasma 198, 1-8 http://dx.doi.org/10.1007/BF01282125

Boyer J. S. (2009): Cell wall biosynthesis and the molecular mechanism of plant enlargement. Funct. Plant Biol. 36, 383-394 http://dx.doi.org/10.1071/FP09048

Cardenas L., McKenna S. T., Kunkel J. G., Hepler P. K. (2006): $\mathrm{NAD}(\mathrm{P}) \mathrm{H}$ oscillates in pollen tubes and is correlated with tip growth. Plant Physiol. 142, 1460-1468 http://dx.doi.org/10.1104/pp.106.087882

Cardenas L., Lovy-Wheeler A., Kunkel J. G., Hepler P. K. (2008): Pollen tube growth oscillations and intercellular calcium levels are reversibly modulated by actin polymerization. Plant Physiol. 146, 1611-1621 http://dx.doi.org/10.1104/pp.107.113035

Chavarria-Krauser A., Yejie D. (2011): A model of plasma membrane flow and cytosis regulation in growing pollen tubes. J. Theor. Biol. 285, 10-24 http://dx.doi.org/10.1016/j.jtbi.2011.06.008

Chebli Y., Kaneda M., Zerzour R., Geitmann A. (2012): The cell wall of the Arabidopsis pollen tube - spatial distribution, recycling, and network formation of polysaccharides. Plant Physiol. 160, 1940-1955 
http://dx.doi.org/10.1104/pp.112.199729

Constantinescu A., Korsunsky A. (2007): Elasticity with Mathematica. An introduction to continuum mechanics and linear elasticity. Cambridge University Press. http://dx.doi.org/10.1017/CBO9780511546167

Cosgrove D. J. (1997): Creeping walls, softening fruit, and penetrating pollen tubes: The growing roles of expansin. Proc. Natl. Acad. Sci. U.S.A. 94, 5504-5505 http://dx.doi.org/10.1073/pnas.94.11.5504

Dardelle F., Lehner A., Ramdani Y., Bardor M., Lerouge P., Driouich A., Mollet J.-C. (2010): Biochemical and immunocytological characterizations of Arabidopsis pollen tube cell wall. Plant Physiol. 153, 1563-1576 http://dx.doi.org/10.1104/pp.110.158881

Derksen J., Li Y.-Q., Knuiman B., Geurts H. (1999): The wall of Pinus sylvestris L. pollen tubes. Protoplasma 208, 26-36 http://dx.doi.org/10.1007/BF01279072

Derksen J., Janssen G. J., Wolters-Arts M., Lichtscheidl I., Adlassnig W., Ovecka M., Doris F., Steer M. (2011): Wall architecture with high porosity is established at the tip and maintained in growing pollen tubes of Nicotiana tabacum. Plant J. 68, 495-506 http://dx.doi.org/10.1111/j.1365-313X.2011.04703.x

Domozych D. S., Fujimoto C., LaRue T. (2013): Polar expansin dynamics in the plant kingdom: A diverse and multifunctional journey on the path to pollen tubes. Plants 2, 148-173 http://dx.doi.org/10.3390/plants2010148

Dumais J., Shaw S. L., Steele C. R., Long S. R., Ray P. M. (2006): An anisotropic-viscoplastic model of plant cell morphogenesis by tip growth. Int. J. Develop. Biol. 50, 209-222 http://dx.doi.org/10.1387/ijdb.052066jd

Eggen E., Keijzer M. N., Mulder B. (2011): Self-regulation in tip-growth: The role of cell wall ageing. J. Theor. Biol. 283, $113-121$ http://dx.doi.org/10.1016/j.jtbi.2011.05.034

Erickson R. O. (1959): Patterns of cell growth and differentiation in plants. In: The Cell. Biochemistry, Physiology, Morphology. pp. 497-535, New York, Academic Press

Erickson R. O. (1986): Symplastic growth and symplasmic transport. Plant Physiol. 82, 1153 http://dx.doi.org/10.1104/pp.82.4.1153

Ferguson C., Teeri T. T., Siika-aho M., Read S. M., Bacic A. (1998): Location of cellulose and callose in pollen tubes and grains of Nicotiana tabacum. Planta 3, 452-460 http://dx.doi.org/10.1007/s004250050421

Geitmann A., Li Y. Q., Cresti M. (1995): Ultrastructural immunolocalization of periodic pectin depositions in the cell wall of Nicotiana tabacum pollen tubes. Protoplasma 187, 168-171 http://dx.doi.org/10.1007/BF01280245

Geitmann A., Parre E. (2004): The local cytomechanical properties of growing pollen tubes correspond to the axial distribution of structural cellular elements. Sex. Plant Reprod. 17, 9-16 http://dx.doi.org/10.1007/s00497-004-0210-3

Geitmann A., Ortega J. K. E. (2009): Mechanics and modelling of plant cell growth. Trends Plant Sci. 14, 467-78 http://dx.doi.org/10.1016/j.tplants.2009.07.006

Geitmann A. (2010): Mechanical modelling and structural analysis of the primary plant cell wall. Curr. Opinion Plant Biol. 13, 693-699 http://dx.doi.org/10.1016/j.pbi.2010.09.017

Haduch-Sendecka A., Pietruszka M., Zajdel P. (2014): Power spectrum, growth velocities and cross-correlations of longitudinal and transverse oscillations of individual Nicotiana tabacum pollen tube. Planta 240, 263-276 http://dx.doi.org/10.1007/s00425-014-2083-5

Harris Ch. M. (1998): The Fourier analysis of biological transients. J. Neurosci. Meth. 83, 15-34

Hayashi T. (1989): Xyloglucans in the primary cell wall. Annu. Rev. Plant Physiol. Plant Mol. Biol. 40, 139-168 http://dx.doi.org/10.1146/annurev.pp.40.060189.001035

Hayashi T., Ogawa K., Mitsuishi Y. (1994): Characterization of the adsorption of xyloglucan to cellulose. Plant Cell Physiol. 35, 1199-1205

Heslop-Harrison J. (1987): Pollen germination and pollen-tube growth. Int. Rev. Cytol. 107, 1-78

http://dx.doi.org/10.1016/S0074-7696(08)61072-4

Hill A. E., Shachar-Hill B., Skepper J. N., Powell J., Shachar-Hill Y. (2012): An osmotic model of the growing pollen tube. PLoS ONE 7, e36585 http://dx.doi.org/10.1371/journal.pone.0036585

Holdaway-Clarke T. L., Hepler P. K. (2003): Control of pollen tube growth: role of ion gradients and fluxes. New Phytol. 159, 539-563 http://dx.doi.org/10.1046/j.1469-8137.2003.00847.x

Jauh G. Y., Lord E. M. (1996): Localization of pectins and arabinogalactan-proteins in lily (Lilium longiflorum L.) pollen tube and style, and their possible roles in pollination. Planta 199, 251-261 http://dx.doi.org/10.1007/BF00196566

Koch A. L. (1982): The shape of the hyphal tips of fungi. J. Gen. Microbiol. 128, 947-951 http://dx.doi.org/10.1099/00221287-128-5-947

Kroh M., Knuiman B. (1982): Ultrastructure of cell wall and plugs of tobacco pollen tubes after chemical extraction of polysaccharides. Planta 154, 241-250 http://dx.doi.org/10.1007/BF00387870

Kroeger J. H., Zerzour R., Geitmann A. (2011): Regulator or driving force? The role of turgor pressure in oscillatory plant cell growth. PLoS ONE 6, e18549 http://dx.doi.org/10.1371/journal.pone.0018549

Lancelle S. A., Hepler P. K. (1992): Ultrastructure of freezesubstituted pollen tubes of Lilium longiflorum. Protoplasma 167, 215-230 http://dx.doi.org/10.1007/BF01403385

Lockhart J. A. (1965): An analysis of irreversible plant cell elongation. J. Theor. Biol. 8, 264-275 http://dx.doi.org/10.1016/0022-5193(65)90077-9

Marzec M., Melzer M., Szarejko I. (2013): Asymmetric growth of root epidermal cells is related to the differentiation of root hair cells in Hordeum vulgare (L.). J. Exp. Bot. 64, $5145-5155$

http://dx.doi.org/10.1093/jxb/ert300

McKenna S. T., Kunkel J. G., Bosch M., Rounds C. M., Vidali L., Winship L. J., Hepler P. K. (2009): Exocytosis precedes and predicts the increase in growth in oscillating pollen tubes. Plant Cell 21, 3026-3040 http://dx.doi.org/10.1105/tpc.109.069260 
Messerli M. A., Robinson K. R. (2003): Ionic and osmotic disruptions of the lily pollen tube oscillator: testing proposed models. Planta 217, 147-157

Ortega J. K. E. (1985): Augmented growth equation for cell wall expansion. Plant Physiol. 79, 318-320 http://dx.doi.org/10.1104/pp.79.1.318

Parre E., Geitmann A. (2005): Pectin and the role of the physical properties of the cell wall in pollen tube growth of Solanum chacoense. Planta 220, 582-592 http://dx.doi.org/10.1007/s00425-004-1368-5

Panovko Y. G., Gubanova I. I. (1965): Stability and Oscillations of Elastic Systems. pp. 84-88, Consultants Bureau, New York

Pietruszka M. (2010): Exact analytic solutions for a global equation of plant cell growth. J. Theor. Biol. 264, 457-466 http://dx.doi.org/10.1016/j.jtbi.2010.02.012

Pietruszka M. (2012): A biosynthesis/inactivation model for enzymatic WLFs or non-enzymatically mediated cell evolution. J. Theor. Biol. 315, 19-127 http://dx.doi.org/10.1016/j.jtbi.2012.09.016

Pietruszka M. (2013): Pressure - induced cell wall instability and growth oscillations in pollen tubes. PLoS ONE 8, e75803. http://dx.doi.org/10.1371/journal.pone.0075803

Plyushch T. A., Willemse M. T. M., Franssen-Verheijen M. A. W., Reinders M. C. (1995): Structural aspects of in vitro pollen tube growth and micropylar penetration in Gasteria verrucosa (Mill.) H. Duval and Lilium longiflorum Thunb. Protoplasma 187, 13-21 http://dx.doi.org/10.1007/BF01280228

Proseus T. E., Boyer J. S. (2007): Tension required for pectate chemistry to control growth in Chara corallina. J. Exp. Bot. $\mathbf{5 7}, 3989-4002$ http://dx.doi.org/10.1093/jxb/erl166

Proseus T. E., Boyer J. S. (2008): Calcium pectate chemistry causes growth to be stored in chara corallina: a test of the pectate cycle. Plant Cell. Environ. 31, 1147-55 http://dx.doi.org/10.1111/j.1365-3040.2008.01829.x

Rojas E. R., Hotton S., Dumais J. (2011): Chemically mediated mechanical expansion of the pollen tube cell wall. Biophys. J. 101, 1844-1853 http://dx.doi.org/10.1016/j.bpj.2011.08.016

Schlüpmann H., Bacic A., Read S. M. (1994): Uridine diphosphate glucose metabolism and callose synthesis in cultured pollen tubes of Nicotiana alata Link et Otto. Plant Physiol. 105, 659-670
Taiz L. (1984): Plant cell expansion: Regulation of cell wall mechanical properties. Ann. Rev. Plant Physiol. 35, 585-657 http://dx.doi.org/10.1146/annurev.pp.35.060184.003101

Taiz L., Zeiger E. (2006): Plant Physiology. 4th edition, Sinauer Associates, Inc., Veytsman B. A., Cosgrove D. J. (1998): A model of cell wall expansion based on thermodynamics of polymer networks. Biophys. J. 75, 2240-2250

Wei C., Lintilhac P. (2003): Loss of stability - a new model for stress relaxation in plant cell walls. J. Theor. Biol. 224, 305-312 http://dx.doi.org/10.1016/S0022-5193(03)00167-X

Wei C., Lintilhac P. M. (2007): Loss of stability: A new look at the physics of cell wall behaviour during plant cell growth. J. Theor. Biol. 283, 113-121

Winship L. J., Obermayer G., Geitmann A., Hepler P. K. (2010): Under pressure, cell walls set the pace. Trends Plant Sci. 15, 363-369 http://dx.doi.org/10.1016/j.tplants.2010.04.005

Winship L. J., Obermayer G., Geitmann A., Hepler P. K. (2011): Pollen tubes and the physical world. Trends Plant Sci. 16, 353-355 http://dx.doi.org/10.1016/j.tplants.2011.03.010

Zerzour R., Kroeger J., Geitmann A. (2009): Polar growth in pollen tubes is associated with spatially confined dynamic changes in cell mechanical properties. Dev. Biol. 334, 437-446 http://dx.doi.org/10.1016/j.ydbio.2009.07.044

Zonia L., Müller M., Munnik T. (2006): Hydrodynamics and cell volume oscillations in the pollen tube apical region are integral components of the biomechanics of Nicotiana tabacum pollen tube growth. Cell Biochem. Biophys. 46, 209-232 http://dx.doi.org/10.1385/CBB:46:3:209

Zonia L., Munnik T. (2008): Vesicle trafficking dynamics and visualization of zones of exocytosis and endocytosis in tobacco pollen tubes. J. Exp. Bot. 59, 861- 873 http://dx.doi.org/10.1093/jxb/ern007

Zonia L., Munnik T. (2011): Understanding pollen tube growth: the hydrodynamic model versus the cell wall model. Trends Plant Sci. 16, 347-352

http://dx.doi.org/10.1016/j.tplants.2011.03.009

Received: September 29, 2014

Final version accepted: November 3, 2014

First published online: March 2, 2015 